\title{
Candidate SNP markers of rheumatoid arthritis changing the affinity of TATA-binding protein for the human gene promoters expo disruptive selection of immunoactivative and immunosuppressive genenets that provoke and prevent this disorder, respectively, as if it could be a self-domestication syndrome
}

Natalya Klimova

Molecular Genetics Department ICG SB RAS

Novosibirsk, Russia

klimova@bionet.nsc.ru

\author{
Dmitry Oshchepkov \\ Systems Biology Department \\ ICG SB RAS \\ Novosibirsk, Russia \\ diman@bionet.nsc.ru
}

\author{
Irina Chadaeva \\ Systems Biology Department \\ ICG SB RAS \\ Novosibirsk, Russia \\ ichadaeva@bionet.nsc.ru \\ Mikhail Ponomarenko \\ Systems Biology Department \\ ICG SB RAS \\ Novosibirsk, Russia \\ pon@bionen.nsc.ru
}

\author{
Evgeniya Oshchepkova \\ Systems Biology Department \\ ICG SB RAS, Novosibirsk, Russia \\ nzhenia@bionet.nsc.ru \\ Academician Vladimir Kozlov \\ RIFCI SB RAS \\ Novosibirsk, Russia \\ niiki01@online.nsk.su
}

\begin{abstract}
Here we conducted a computational genome-wide study of the all known single-nucleotide polymorphism (SNP) of 70 bp proximal promoters of 67 human rheumatoid arthritis (RA)-related genes that displayed disruptive natural selections of immunoactivative or immunosuppressive genes raising or reducing risks of $\mathrm{RA}$, respectively, as if it maybe a domestication syndrome. That is why, we confirmed it in vivo using the genomewide transcriptome profiling (RNA-seq assay) of the differentially expressed genes (DEGs) within hypothalamus of adult male rats (Rattus norvegicus) of two unique outbred lines bred in aggressiveness and tameness as an animal model of human diseases (statistical significance padj $<0.025$ at Pearson's $\chi^{2}$ criterion with Bonferroni's correction).
\end{abstract}

Keywords - rheumatoid arthritis, human, gene, promoter, TATA-binding protein (TBP) binding site (TATA box), single nucleotide polymorphism (SNP), candidate SNP marker, verification

\section{Background}

Rheumatoid polyarthritis (RA) is an autoimmune disease with autoantibodies (e.g., antibodies to citrullant antigens) and proinflammatory cytokines (e.g., TNF- $\alpha$, IL-6) that are involved in the induction of chronic synovitis, bone erosion, followed by deformity [1]. Currently, it is commonly accepted that immunopathogenesis is mostly contributed by the mechanisms of the breakdown of immune tolerance to its own antigens that is characterized by an increase in the activity of T-effector cells, causing RA symptoms [2]. On the contrary, a low activity of regulatory cells (e.g., regulatory T-cells (Treg) and myeloid suppressor cells) is met in RA [3]. That is why, to say that it is the immunosuppressor cell deficit that is the central feature in RA pathogenesis [4]. It is thought that lifestyle and living conditions define a half of the RA risks and genetic susceptibility to RA do for another half [5]. Additionally, immune cells of adaptive memory in a given individual keep information on all diseases he / she survived that increase his / her resistance to them in future [6]. That is why here using our SNP TATA_Comparator [7], we predicted RA-related candidate SNP markers, found regularities in their genome-wide frequencies, and tested them in vivo using an animal (rat) model of the human health.

\section{Materials and Methods}

We extracted 1834 SNPs of 70 bp promoters of 67 RArelated genes using UCSC Genome Browser [8], dbSNP [9] and GRCh38/hg38 assembly of the human reference genome [10] as well as treated them using our SNP_TATA_Comparator [7] as discribed elsewhere [7]. We did the experiment in vivo on 6 adult male rats (Rattus norvegicus) of 2 outbred lines bred (over 90 generations) for aggressive or tame behavior under standard conditions of the Conventional Animal Facility of ICG SB RAS (Novosibirsk, Russia), as described elsewhere [11]. All rats came from different litters and were unrelated, each weighing 250-270 g, 4 months old, marked by "-3" or " +3 " on the rank scale from "$4 "$ to "+4" (i.e., maximum aggressiveness and tameness). After their euthanasia, the biosamples of hypothalamus was prepared and stored at $-70{ }^{\circ} \mathrm{C}$ until use as approved by Interinstitutional Bioethics Board at ICG SB RAS in line with Directive 2010/63/EU of the European Parliament and of the Council of September 22, 2010, on protections of animals used for scientific needs, as described in [12].With $100 \mathrm{mg}$ fractions taken from each above-mentioned hypothalamic biosample from three aggressive and three manual rats, using an Illumina NextSeq 550 and NextSeq ${ }^{\circledR}$ 500/550 High Output Kit v2 (75 cycles) cassettes, we sequenced through direct reading 24 barcoded RNA-Seq libraries of $75 \mathrm{nt}$ fragments that lead the total volume of 400 million reads. We processed them by our conveyor, as: FastQC [13] $\rightarrow$ Trimmomatic [14] $\rightarrow$ TopHat2 $[15] \rightarrow$ SAMtools [16] $\rightarrow$ HTSeq [17] $\rightarrow$ DeSeq2 [18]. This resulted in relative expression of genes mapped into the rat reference genome rn6 in hypothalamus of tame and aggressive rats. We verified them with our measurements of them made by quantitative polymerase chain reaction (qPCR) in our work [19]. Finally, among 67 genes studied, we found those whose expression were significantly different between aggressive and tame rats, evaluated the manifestations of them in terms of susceptibility to RA according to the clinical markers of RA 
and, estimated the statistical significance of them with Bonferroni correction.

\section{Results and Discussions}

First, using the only clinical SNP marker s2276109 of RA [20], we tested adequacy of candidate SNP markers of this disease predicted by SNP TATA Comparator [9] Then, for 646 SNPs in question within $2 \overline{7}$ human genes clinically associated with disorders comorbid to RA, we found 83 and 73 candidate SNP markers, which can elevate (e.g., rs1185314734) or reduce (e.g., rs1190659847) expression of these genes, respectively, as well as 91 and 54 ones for the worsened (e.g., rs1189849606) or relieved (e.g., rs549858786) RA. It means the genome-wide neutral drift [21-23] towards susceptibility to RA in line with the common view that diversity of adaptive memory immune cells of a given individual grow with each inflammation he/she survived that improves his/her resistance to them, but this immune memory growth raises risks of impaired autoimmune tolerance (e.g., RA risks). Thus, by the same way we confirmed this finding in the case of nine genes, which are most GWAS-associated with RA [24], and, independently, in the case of seven immunoactivative genes. Cntrary, when we dealt with 24 immunosuppressive genes, we found 64 and 111 candidate SNP markers aggravating and relieving RA, respectively. This difference between immunoactivative and immunosuppressive genes means disruptive natural selection as if RA could be a domestication syndrome. That is why, using RNA-seq assay we compared aggressive and tame rats, both primary experimental and resulting computational data of which are available for readers, as: "ftp://ftp.RNAseq.cytogen.ru:h3\&3r" using "login/password: Ht3\&3Rats". It turned out that 10 among 67 genes analyzed here are differentially expressed in hypothalamus of adult male aggressive versus domesticated rats at partial significance threshold $p_{R N A s e q}<0.05$. For four genes Apoal, Hbb, Hbb-b1, and Sod1, it is known that overand underexpression of them worsen RA, while six rest genes Ccr7, Ebi3, Illb, Il2rb, Il3ra, and Il25 overexpress in tame rats in line with the physiological markers of the worsen RA. Within Pearson's $\chi^{2}$-criterion, it is susceptibility to RA in the tame rats $\left(\mathrm{p}_{\chi 2}<0.005\right)$. So, $\mathrm{p}_{\mathrm{adj}}=\mathrm{N} * \mathrm{p}_{\chi 2} * \mathrm{p}_{\mathrm{RNAseq}}=67 * 0.005 * 0.05$ $<0.025$, as Bonferroni's correction.

\section{Conclusions}

We found in silico disruptive selections of immunoactivative or immunosuppressive genes corresponding high or low risks of RA, as if it could be a domestication syndrome, which we confirmed at animal model of human health..

\section{ACKNOWLEDGMENTS}

Manuscript writing was supported by project \#0324-20190040-C-01 (for MP). The experiment in vivo was supported by project \#0324-2019-0042-C-01 (for NK, IC). The data analysis and biological interpretation were supported by the Russian Federal Science \& Technology Program for the Development of Genetic Technologies (for DO, EO).

\section{REFERENCES}

[1] J. Smolen, D. Aletaha, Nat Rev Rheumatol, vol. 11, pp. 276-289, 2015.

[2] J. Sarkander et al., Clin Transl Immunology, vol. 5, pp. e120, . 2016.

[3] O. Alsaed et al., Eur J Case Rep Intern Med, vol. 5, pp. 000895, 2018.

[4] C. Lawson et al., Rheumatology (Oxford), vol. 45, pp. 1210-1217, 2006.

[5] N. Nair et al., Pharmacogenomics, vol. 18, pp. 1323-1332, . 2017.

[6] J. Sarkander, et al., Clin Transl Immunology, vol 5, pp. e120, . 2016.

[7] M. Ponomarenko et al.. Biomed. Res. Int. vol. 2015. pp. 35983004625. 2015.

[8] M. Haeussler et al., Bioinformatics. vol. 31. pp. 764-766. 2015..

[9] S. Sherry et al., Nucleic Acids Res. vol. 29, pp. 308-311, 2001.

[10] D. Zerbino et al., Genome Biol. vol. 16: pp. 56. 2015.

[11] D. Belyaev, P. Borodin, Biol Zent B1, vol. 100, pp. 705-714, 1982.

[12] I. Plyusnina, I. Oskina, Physiol Behav, vol. 61, pp. 381-385, 1997.

[13] K. Kroll et al., Cancer Inform, vol. 13(Suppl 3), pp. 7-14, 2014.

[14] A. Bolger et al., Bioinformatics, vol. 30, pp. 2114-2120, 2014.

[15] D. Kim et al., Genome Biol, vol. 14, pp. R36, 2013.

[16] Li H. Bioinformatics, vol. 27, pp. 1157-1158, 2011.

[17] S. Anders et al., Bioinformatics, vol. 31, pp. 166-169, 2015.

[18] M. Love et al., Genome Biol, vol. 15, pp. 550, 2014.

[19] D. Oshchepkov et al., Front Genet,vol.. 10, pp. 1267, 2019.

[20] G. Hunninghake et al., N Engl J Med, vol. 361, pp. 2599-2608, . 2009

[21] J. Haldane. J Genet. vol. 55. pp. 511-524. 1957.

[22] M. Kimura, Nature. vol. 217. pp. 624-626. 1968.

[23] 1000 Genomes Project Consortium, "Nature. vol. 491. pp. 56-65. 2012.

[24] J. Karami et al., Gene, vol. 702, pp. 8-16, 2019. 